DOI: $10.2507 / 27$ th. daaam.proceedings. 046

\title{
METHOD OF FAULT IDENTIFICATION IN MECHATRONIC SYSTEMS
}

\author{
Vladimir Filaretov, Alexey Zhirabok, Alexander Zuev, Alexander Protsenko
}
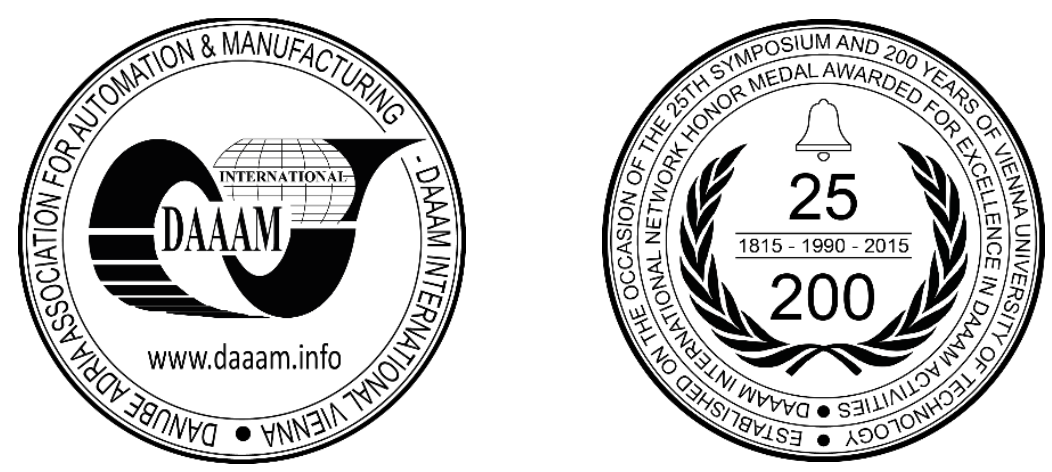

This Publication has to be referred as: Filaretov, V[ladimir]; Zhirabok, A[lexey]; Zuev, A[lexander] \& Protsenko, A[lexander] (2016). Method of Fault Identification in Mechatronic Systems, Proceedings of the 27th DAAAM International Symposium, pp.0312-0318, B. Katalinic (Ed.), Published by DAAAM International, ISBN 978-3-90273408-2, ISSN 1726-9679, Vienna, Austria

DOI: $10.2507 / 27$ th.daaam.proceedings.046

\begin{abstract}
This paper considers amethod of synthesis of identification system for faults appearing in the various elements of complex mechatronic systems described by nonlinear differential equations. To solve this problem logic-dynamic approach and special feedback by residual signal is suggested. This approach consists of three main steps: replacing the initial nonlinear system by certain linear logic-dynamic system, obtaining the bank of linear logic-dynamic observers, and transforming these observes into the nonlinear ones. Logic-dynamic approach allows detecting and isolating faults in nonlinear dynamic systems by using the linear methods for diagnosis in nonlinear mechatronic systems. Feedback of special formintroduced in the observers after the detection and isolation of faults. It is allowing to determine the value of faults in nonlinear systems with incomplete measurability of state vector. Efficiency and high performance of the proposed method is shown on example of identification of faults arising in electric drives of multilink manipulator.
\end{abstract}

Keywords: Fault identification; diagnostic; observers; mechatronic systems; nonlinear systems.

\section{Introduction}

Operative detection and localization of faults that may occur in elements and subsystems in the process of operation it is one of the perspective ways to improve the efficiency of operation of mechatronic systems(MS) in the event of minor defects in the sensor elements and actuators. Timely detection, localization and identification of the values of these faults allow the use of methods of fault-tolerant control and accommodation for the formation of specific control signals that allow to keep the most important characteristics of the system in the presence of faults in it providing guaranteed performance of specified operations and missions.

Currently, there are several different approaches to the synthesis of the system of identification of faults [1-9]. All these approaches are based on the procedures for the real-time identification and localization of faults via diagnostic observers (DO). Several methods for synthesis of diagnostic observers for nonlinear dynamical systems are known, including those based on the differential geometric approach [5], the algebra of functions [5], logic-dynamic approach [1$3,9]$ and others [4, $6-8]$. The first two allow obtaining the optimal solution with a minimum dimension of observer, however, have a rather complicated process of finding a solution, because of complex analytical calculations. Logicdynamic approach does not guarantee a minimum dimension, but the process of finding a solution on its basis is quite 
simple because of using of linear methods. The task of identifying the values of the faults on the basis of logic-dynamic approach has been solved in [2] and [3], by introducing into the diagnostic observers the signal of feedback by the residual. However, this approach is valid only for the case when the state vector of the diagnosing object fully measurable and diagnostic observers has the first order.

This paper considers solution to the problem of developing a method of synthesis of precise identification system for faults in a variety of complex MS, which use the DO, described by differential equations with the order above the first. In method a special feedback by the signal of residual is introduced in the diagnostic observers obtained with the logicdynamic approach for real-time identification of values of faults.

\section{Model of diagnosed object}

In general, non-linear multidimensional diagnosed object (DO) can be described by a model of the form:

$$
\begin{aligned}
& \dot{x}(t)=F x(t)+B(x(t), u(t))+G u(t)+L d(t), \\
& y(t)=H x(t),
\end{aligned}
$$

where $x \in R^{n}$ is state vector, $n$ is dimensionality of DO; $y \in R^{m}$ is output vector; $u \in R^{l}$ is input vector, $p$-dimensional vector $d(t)$ describes the errors occurring in the system because of the appearance of faults in it (in the absence of faults all the elements of the vector are zero; in the presence of a fault corresponding element of the vector becomes unknown function of time); $F \in R^{n \times n}$ is matrix of system dynamics; $G \in R^{n \times l}$ is matrix that determines the effect of the input to the system; $H \in R^{m \times n}$ is matrix that determines the relationship of output with the state vector; $B(x(t), u(t))$ is a vector which determines a non-linear part of the system. In this paper a case where each of elements of vector $d(t)$ included only in one of the equations of (1) is considered, which means that there is only one nonzero element in each of the columns of matrix $L \in R^{n \times p}$. The task of detecting faults and determining their values are invited to decide on the basis of logicdynamic approach (LDA) [1], which allows the construction of diagnostic observers using only linear methods. After synthesis of the diagnostic observers, the special feedback must be introduced in them to ensure stability and to determine the value of the faults occurring in the system.

The developed method provides a set of nonlinear diagnostic observers, each of which is sensitive to one of the faults and invariant to the others. The output signals of observers are the values of the corresponding faults.

As a result of the well-known procedure of synthesis of diagnostic observers based on the LDA [1] $k$-dimensional observer sensitive to one of the faults $d_{i}(t), i=\overline{1, p}$, can be built in the following form:

$$
\begin{aligned}
& \dot{x}^{*}(t)=F^{*} x^{*}(t)+B^{*}\left(x^{*}(t), u(t)\right)+G^{*} u(t)+J y(t), \\
& y^{*}(t)=H^{*} x^{*}(t),
\end{aligned}
$$

where $\mathrm{x} \in R^{k}$ is state vector of observer; $y^{*}$ is output signal of observer; $F^{*} \in R^{k \times k}, G^{*} \in R^{k \times l}, H^{*} \in R^{1 \times n}$ are matrixes corresponding to matrices of system (1), $J \in R^{k \times m}$ is matrix, which defines the use of DO output in observer. All these matrices must be determined during the synthesis of the observer. It is known [1] that $F^{*}$ и $H^{*}$ can be chosen as:

$$
F^{*}=\left[\begin{array}{ccccc}
0 & 1 & 0 & \ldots & 0 \\
0 & 0 & 1 & \ldots & 0 \\
\ldots & \ldots & \ldots & \ldots & \ldots \\
0 & 0 & 0 & \ldots & 0
\end{array}\right], H^{*}=\left[\begin{array}{lllll}
1 & 0 & 0 & \ldots & 0
\end{array}\right] .
$$

On the basis of the outputs of DO and observer following residual is generated:

$$
r(t)=C \mathrm{y}(t)-\mathrm{y}^{*}(t)
$$

where $C$ is $m$-dimensional vector, defining use of DO output in residual generation.

The state vectors of DO and observer are associated by the matrix $\Phi \in R^{k \times n}$, and, in the absence of a mismatch between the DO and the observer (if $r=0$ ), satisfy the following equations[1]:

$$
x^{*}=\Phi x, G^{*}=\Phi G, C H=H^{*} \Phi, \Phi F=F^{*} \Phi+J H
$$


In case of any fault the residual (4) becomes different from zero, and the equations (5) is no longer fulfilled. To solve the problem of identification of faults, consider how residual will change in the event of faults. After differentiating the expression (4) taking into account (1) and (2) it can be obtained:

$$
\dot{r}=C H(F x+G u+B(x, u)+L d)-H^{*}\left(F^{*} x^{*}+G^{*} u+B^{*}\left(x^{*}, u\right)+J y\right)
$$

In view of this and (5) it can be obtained:

$$
\dot{r}=H^{*}\left(F^{*}\left(\Phi x-x^{*}\right)+\Phi B(x, u)-B^{*}\left(x^{*}, u\right)+\Phi L d\right)
$$

Denote the value of $\Phi x-x^{*}$ as a vector $e \in R^{k}$ of mismatch of states of DO and the observer: $e=\Phi x-x^{*}$. Considering introduced vector, it can be obtained:

$$
\dot{r}=H^{*}\left(F^{*} e+\Phi B(x, u)-B^{*}\left(x^{*}, u\right)+\Phi L d\right)
$$

Consider the relationship of the vector of mismatch $e$ with the residual $r$, in view of (4):

$$
r=C y-y^{*}=C H x-H^{*} x^{*}=H^{*} \Phi x-H^{*} x^{*}=H^{*} e .
$$

Thus, taking into account the form of vector $H^{*}$, the residual $r$ is the first element of mismatch vector $e$ :

$$
r=e_{1}
$$

Next, consider the impact of a fault not only on residual, but on the entire vector $e$. Then, taking into account (5) and (7) it can be obtained:

$$
\dot{e}=F^{*} e+\Phi B(x(t), u(t))-B^{*}\left(x^{*}(t), u(t)\right)+\Phi L d
$$

From (8) it follows that the change of the mismatch vector depends on the element $\Phi B(x(t), u(t))-B^{*}\left(x^{*}(t), u(t)\right)$, which characterizes the non-linearity of DO and observer. In the case of incomplete measurability of DO, state vector $x$ and the value of the element $\Phi B(\mathrm{x}(t), \mathrm{u}(t))$ can be unknown. It is making difficult identifying of value of faults. In LDA observer constructed in way to compensate the effect of non-linearity in the residual signal and $\Phi B-B^{*}=0$. However, this condition is fulfilled only when there is no mismatch between the state vectors of DO and observer and $e=0$. To determine the value of fault $d_{i}$, which we are interested, it is necessary to introduce feedback by residual signal formed in such way to provide mismatch elimination even in the case of faults.

\section{Introduction of feedback}

After the introduction of feedback by residual signal in the observer, its model assumes the form:

$$
\begin{aligned}
& \dot{x}^{*}(t)=F^{*} x^{*}(t)+B^{*}\left(x^{*}(t), u(t)\right)+G^{*} u(t)+w(r)+J y(t), \\
& y^{*}(t)=H^{*} x^{*}(t)
\end{aligned}
$$

where $w(r) \in R^{k}$ is a vector specifying feedback by residual signal.

Taking into account the introduced feedback, change of the mismatch vector will be determined by the equation:

$$
\dot{e}=F^{*} e+\Phi B(x, u)-B^{*}\left(x^{*}, u\right)+\Phi L d-w(r) .
$$

If formed feedback ensures that the equalities (4) and $B^{*}(x(t), u(t))=\Phi B\left(x^{*}(t), u(t)\right)$ are performed, nonlinearity in the process of identification of faults will be compensated.

To ensure the sensitivity of observer to the fault $d_{i}$ and the invariance of the rest, matrix $\Phi$ is constructed so that all the columns of matrix $\Phi L$ except $i$ th were zero, so observer will be affected only by one element of fault vector $d$. Next, consider common case, when the equation (11) can be represented as: 


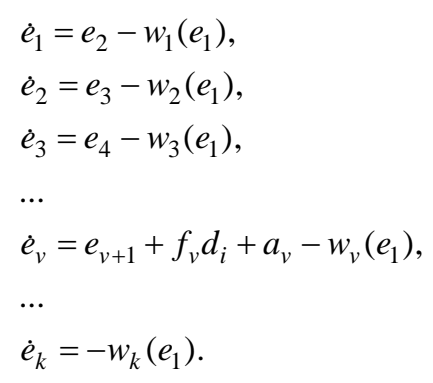

where $a=\Phi B(x(t), u(t))-B^{*}\left(x^{*}(t), u(t)\right), f_{v}$ is element of the $i$ th column of the matrix $\Phi L$.

In this case, the fault and uncompensated nonlinear component $a_{v}$ are present in only one of the equations (11) In the case of $w_{v+1}\left(e_{1}\right)=0, \ldots, w_{k}\left(e_{1}\right)=0$ and the consistency of the initial conditions of DO and observer, $e_{v+1}, \ldots, e_{k}$ are zero regardless of the occurrence of the fault. Then further, it is possible to consider only a part of the system of equations (11):

$$
\begin{aligned}
& \dot{e}_{1}=e_{2}-w_{1}\left(e_{1}\right), \\
& \dot{e}_{2}=e_{3}-w_{2}\left(e_{1}\right), \\
& \dot{e}_{3}=e_{4}-w_{3}\left(e_{1}\right), \\
& \ldots \\
& \dot{e}_{v}=f_{v} d_{i}+a_{v}-w_{v}\left(e_{1}\right) .
\end{aligned}
$$

Writing the system of equations (13) in the form of a differential equation we get:

$$
f_{v} d_{i}+a_{v}=\dot{e}_{v}+w_{v}=\ddot{e}_{v-1}+\dot{w}_{v-1}+w_{v}=\dddot{e}_{v-2}+\ddot{w}_{v-2}+\dot{w}_{v-1}+w_{v}=e_{1}^{(v)}+w_{1}^{(v-1)}+\ldots+w_{v-1}^{(1)}+w_{v} .
$$

Accepting elements of the vector of feedback, except the $v$ th, proportional to the residual $w_{j}=\frac{T_{i}}{T_{0}} e_{1}(j=\overline{1, v-1})$ and $w_{v}=\frac{T_{v}}{T_{0}} e_{1}+\frac{1}{T_{0}} z\left(e_{1}\right)$, it can be obtained:

$$
f_{v} d_{i}+a_{v}=e_{1}^{(v)}+\frac{T_{1}}{T_{0}} e_{1}^{(v-1)}+\ldots+\frac{T_{v-1}}{T_{0}} e_{1}^{(1)}+\frac{T_{v}}{T_{0}} e_{1}+\frac{1}{T_{0}} z\left(e_{1}\right)
$$

If value of $f_{v} d_{i}+a_{v}$ presented as input signal, and $e_{1}$ as output, equation(14) can be represented as a transfer function:

$$
W=\frac{T_{0}}{T_{0} s^{v}+\ldots+T_{v}+Z(s)} .
$$

Suppose that input $f_{v} d_{i}+a_{v}$ can be represented as a polynomial of degree $q: f_{v} d_{i}+a_{v}=b_{0}+b_{1} t+\ldots+b_{q} t^{q}$. The Laplace transform of such input is $Q=\frac{b_{0}}{s}+\frac{b_{1}}{s^{2}}+\ldots+\frac{b_{q}}{s^{q+1}}$.

Then the steady value of $e_{1}$ can be found as: $e_{1}=\lim _{s \rightarrow 0} W Q s=\lim _{s \rightarrow 0} \frac{T_{0}\left(b_{0} s^{q-1}+\ldots+b_{q}\right) s}{\left(T_{0} s^{v}+\ldots+T_{v}\right) s^{q+1}+Z(s) s^{q+1}}$. Assuming $Z(s)=\frac{T_{v+1}}{s}+\frac{T_{v+2}}{s^{2}}+\ldots+\frac{T_{v+q}}{s^{q}}+\frac{T_{v+q+1}}{s^{q+1}}$ we get: $e_{1}=\lim _{s \rightarrow 0} \frac{T_{0}\left(b_{0} s^{q-1}+\ldots+b_{q}\right) s}{\left(T_{0} s^{v}+\ldots+T_{v}\right) s^{q+1}+T_{v+1} s^{q}+\ldots+T_{v+q+1}}=0$.

Thus introducing a feedback of indicated type result in a steady value of $e_{1}=0$. This also results in resetting of all elements of vector $e$. For many real systems value of the fault is constant or slowly changing and it is sufficient to introduce one integral of residual in feedback to achieve the required accuracy of the identification of faults:

$$
z\left(e_{1}\right)=\int e_{1} d t
$$


Thus, after the introduction of this kind of feedback, residual $e_{1}$ will tend to zero, after the completion of the transition process, including in the event of faults. This will ensure the synchronization of state vectors of DO and observer.

The next step of solving the problem is estimation of value of the desired fault. Since the introduction of the feedback was able to arrange that $e=0$, taking into account (12) it can be written:

$$
d_{i}=\frac{w_{v}\left(e_{1}\right)}{f_{v}} \text { or } \quad d_{i}=\frac{1}{f_{v} T_{0}} \int e_{1} d t
$$

Thus the output of the system of identification of faults after the of transient processes will be sought value $d_{i}$. The required system performance can be achieved by selection of $T_{0} \ldots T_{\nu}$ coefficients.

\section{Example}

Let us to consider multilink manipulator(MM) with $n$ degrees of freedom which is driven by $n$ actuators with DC motors. Each actuator has sensors of the position, speed and current. It is assumed that the following types of faults are possible in the actuators of MM: $d_{1}$ - fault caused by an increase of moment of viscous friction in the link of manipulator; $d_{2}$-fault caused by an increase of moment of viscous friction in the actuator; $d_{3}$ - fault caused by the change of active resistance of rotor circuit of DC motors (for example, in case of significant changing of temperature). The presence of these faults significantly reduces the quality of the actuators and accuracy of performance of specific technological operations.

In the presence of these faults, each actuator of MM without feedback can be described by the nonlinear differential equations in form (1) where:

$$
F=\left[\begin{array}{ccccc}
0 & 1 & 0 & 0 & 0 \\
-c i^{2} / H & -\left(k_{v n}+h\right) / H & c i / H & 0 & 0 \\
0 & 0 & 0 & 1 & 0 \\
c i / J & 0 & -c / J & -k_{v d} / J & k_{\mathrm{m}} / J \\
0 & 0 & 0 & -k_{\omega} / L & -R / L
\end{array}\right], B=\left[\begin{array}{c}
0 \\
\left(-M_{E}-M_{s t n 0} \operatorname{sign}\left(x_{2}\right)\right) / H \\
0 \\
\left.-M_{s t d 0} \operatorname{sign}\left(x_{4}\right)\right) / J \\
0
\end{array}\right], x=\left[\begin{array}{c}
x_{1} \\
x_{2} \\
x_{3} \\
x_{4} \\
x_{5}
\end{array}\right], G=\left[\begin{array}{c}
0 \\
0 \\
0 \\
0 \\
k_{u} / L
\end{array}\right],
$$

$L=\left[\begin{array}{lll}0 & 0 & 0 \\ 1 & 0 & 0 \\ 0 & 0 & 0 \\ 0 & 1 & 0 \\ 0 & 0 & 1\end{array}\right], H=\left[\begin{array}{lllll}1 & 0 & 0 & 0 & 0 \\ 0 & 0 & 0 & 1 & 0 \\ 0 & 0 & 0 & 0 & 1\end{array}\right] d=\left[\begin{array}{c}d_{1} \\ d_{2} \\ d_{3}\end{array}\right]$,

where $R, L, I$ are resistance, inductance and current of DC motor rotor circuits, accordingly; $k_{\omega}$ is a counter-EMF coefficient; $k_{\mathrm{u}}$ is an amplifier coefficients; $k_{m}$ is a moment coefficient; $J$ is a moment of inertia of DC motor shaft and rotating parts of reducers; $c$ is rigidity of mechanical transmission; $x_{1}$ is a turn angle of link; $i$ is a reducing ratio of the reducer; $h, H, M_{E}$ are the variable components of torque influences on actuators, including the interactions between all the degrees of freedom of the manipulator; $u$ is a input voltage of amplifiers.

In this case, the dimension of the output vector of the system less than dimension of state vector Thus, only the value of $x_{1}, x_{4}$ and $x_{5}$ are measured, and $x_{2}$ and $x_{3}$ - no.

Next, consider the synthesis of the identification system for fault $d_{1}$. Diagnostic observer of the third order, which is sensitive to this fault and insensitive to other faults, will be described by system of equations (10) and the following matrix:

$$
F^{*}=\left[\begin{array}{lll}
0 & 1 & 0 \\
0 & 0 & 1 \\
0 & 0 & 0
\end{array}\right], G=\left[\begin{array}{l}
0 \\
0 \\
0
\end{array}\right], J=\left[\begin{array}{ccc}
-\left(k_{v n}+h\right) / H & 0 & 0 \\
-c i^{2} / H & 0 & 0 \\
0 & c i / H & 0
\end{array}\right], C=\left[\begin{array}{lll}
1 & 0 & 0
\end{array}\right], B=\left[-\frac{M_{E}+M_{\operatorname{stn} 0} \operatorname{sign}\left(x_{2}\right)}{H}\right]
$$

Since $\Phi L=\left[\begin{array}{lll}0 & 0 & 0 \\ 1 & 0 & 0 \\ 0 & 0 & 0\end{array}\right]$, the fault $d_{1}$ is affecting only second equation of observer, and feedback vector have the form: $w=\left[\begin{array}{lll}w_{1} & w_{2} & 0\end{array}\right]^{T}$.

Since the value of $x_{2}$, a member of the vector $B$ is not measured by sensors, it must be defined from (4): 
$x_{2}=x_{2}^{*}-\frac{k_{v n}+h}{H} x_{1}^{*}$.

In the synthesis of the third order observer is advisable to select feedback coefficients as follows:

$T_{0}=\left(6 T_{c}\right)^{3}, T_{1}=3\left(6 T_{c}\right)^{2}, T_{2}=18 T_{c}$,

where $T_{c}$ is the time during which the output of the system enters the $5 \%$ area of the value of the desired fault. Accepting $T_{c}=1 s$ we get:

$T_{0}=216, T_{1}=108, T_{2}=18$

Feedback vector in this case will be:

$w=\left[\begin{array}{c}\frac{T_{1}}{T_{0}} e_{1} \\ \frac{T_{2}}{T_{0}} e_{1}+\int \frac{1}{T_{0}} e_{1} d t \\ 0\end{array}\right]$.

To verify the functionality and effectiveness of the proposed method, the modelling of the synthesized faults accommodation system for the model of the actuator of the second degree of freedom $\left(q_{2}\right)$ of PUMA-type manipulator (see Fig.1) was carried out.

At the synthesis of faults accommodation system were used the following parameters of actuator with DC motor: $J=0.0001 \mathrm{~kg} \cdot \mathrm{m} \cdot \mathrm{s}^{2}, k_{\omega}=0.02 \mathrm{~V} \cdot \mathrm{s}, k_{u}=100, R=0.4 \Omega, L=0.004 \mathrm{Hr}, k_{m}=0.02 \mathrm{~N} \cdot \mathrm{m} / \mathrm{A}, i=100, c=2 \mathrm{Nm} / \mathrm{rad}, k_{v n}=10^{-2} \mathrm{~N} \cdot \mathrm{m} \cdot \mathrm{s} / \mathrm{rad}$, $\mathrm{kvd}=10^{-5} \mathrm{~N} \cdot \mathrm{m} \cdot \mathrm{s} / \mathrm{rad}, M_{s t n}=0.06 \mathrm{~N} \cdot \mathrm{m}, \mathrm{M}_{\mathrm{std}}=0.0006 \mathrm{~N} \cdot \mathrm{m}$.

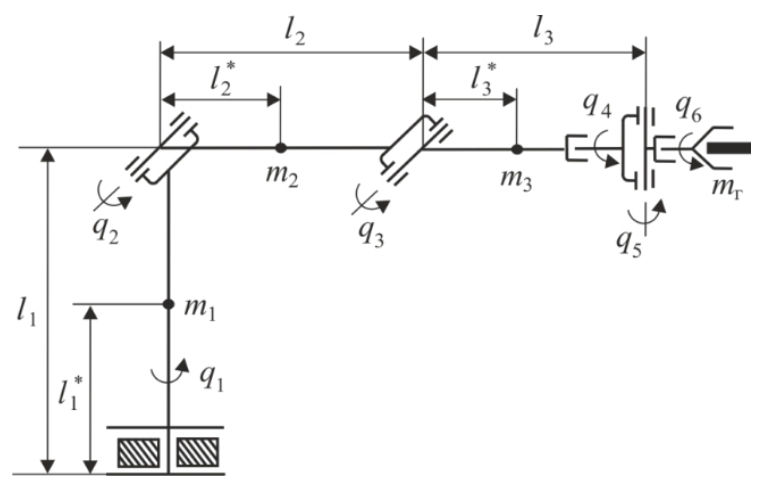

Fig. 1. Kinematical scheme of the manipulator

For the second degree of freedom mutual influence effects in the model are described by the following equations [10]:

$H_{2}=J_{n 2}+J_{n 3}+m_{2} l_{2}^{* 2}+m_{3} l_{3}^{* 2}+\left(m_{3}+m_{\mathrm{g}}\right) l_{2}^{2}+m_{\mathrm{g}} l_{3}^{2}+2 l_{2}\left(m_{3} l_{3}^{*}+m_{\mathrm{g}} l_{3}\right) \cos q_{3}$

$h_{2}=-2 l_{2}\left(m_{3} l_{3}^{*}+m_{\mathrm{g}} l_{3}\right) \dot{q}_{3} \sin q_{3}$,

$M_{\mathrm{E} 2}=\left[J_{n 3}+m_{3} l_{3}^{* 2}+m_{\mathrm{g}} l_{3}^{2}+\left(m_{3} l_{3}^{*}+m_{\mathrm{g}} l_{3}\right) l_{2} \cos q_{3}\right] \ddot{q}_{3}-l_{2}\left(m_{3} l_{3}^{*}+m_{\mathrm{g}} l_{3}\right) \dot{q}_{3}^{2} \sin q_{3}+g\left[m_{2} l_{2}^{*}+\left(m_{3}+m_{\mathrm{g}}\right) l_{2}\right] \sin q_{2}+$

$+g\left(m_{3} l_{3}^{*}+m_{\mathrm{g}} l_{3}\right) \sin \left(q_{2}+q_{3}\right)-\frac{1}{2}\left[\left(J_{n 2}-J_{s 2}+m_{2} l_{2}^{* 2}+\left(m_{3}+m_{\mathrm{g}}\right) l_{2}^{2}\right) \sin 2 q_{2}+\left(J_{n 3}-J_{s 3}+m_{3} l_{3}^{* 2}+m_{\mathrm{g}} l_{3}^{2}\right) \sin 2\left(q_{2}+q_{3}\right)+\right.$

$\left.+2 l_{2}\left(m_{3} l_{3}^{*}+m_{\mathrm{g}} l_{3}\right) \sin \left(2 q_{2}+q_{3}\right)\right] \dot{q}_{1}^{2}$,

where $J_{s i}$ is the moment of inertia of link $i$ about its longitudinal axis; $J_{n i}$ is the moment of inertia of link $i$ of a transverse axis passing through its centre of mass; $g$ is acceleration of gravity.

In modelling the following values of parameters of MM's links were used: $m_{1}=35.8 \mathrm{~kg}, m_{2}=3.5 \mathrm{~kg}, m_{3}=3 \mathrm{~kg}, l_{l}=0.2 \mathrm{~m}$, $l_{2}=0.5 \mathrm{~m}, l_{3}=0.4 \mathrm{~m}, l^{*}{ }_{1}=0.1 \mathrm{~m}, l^{*}{ }_{2}=0.12 \mathrm{~m}, l^{*}{ }_{3}=0.1 \mathrm{~m}, J_{s 2}=0.0047 \mathrm{~kg} \cdot \mathrm{m}^{2}, J_{n 2}=0.12 \mathrm{~kg} \cdot \mathrm{m}^{2}, J_{n 2}=0.067 \mathrm{~kg} \cdot \mathrm{m}^{2}, \mathrm{mg}_{\mathrm{g}}=0.5 \mathrm{~kg}$. 
Faults were simulated by introducing error signals $d_{1}=0.01$ from time $t_{1}=10 \mathrm{~s}$. to $t_{3}=20 \mathrm{~s}$. and $d_{2}=d_{3}=0.1$, from $t_{2}=15 \mathrm{~s}$. Output of observer is shown at Fig. 2.

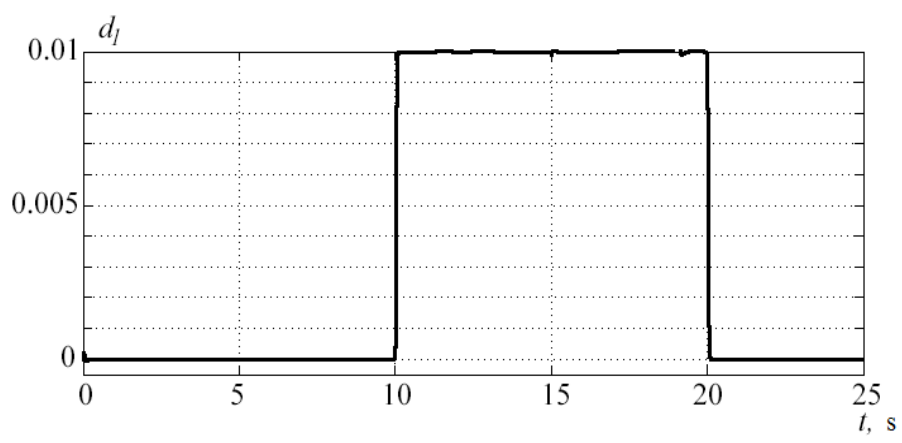

Fig. 2. Output of observer at constant fault value

As can be seen the proposed system accurately determines the presence or absence of a fault $d_{l}$ and its value. In addition, as required, faults $d_{2}$ and $d_{3}$ does not affect the determination of $d_{1}$.

\section{Conclusion}

In this paper method of synthesis of accurate identification systems for occurring faults in a variety of complex MS, which use the DO described by differential equations with the order above the first was developed. This method consists of applying of logic-dynamic approach for synthesis of diagnostic observers, guaranteeing the independence of the detection and localization of possible faults and introducing of special feedback for diagnostic observers, allowing identifying values of faults. Efficiency of the proposed method of synthesis of diagnostic observers for identification of faults in nonlinear dynamic systems was confirmed by the results of mathematical modelling. The subject of further research will be application of the proposed method for synthesis of the fault accommodation and failsafe control systems for such objects as industrial manipulators and underwater vehicles.

\section{Acknowledgments}

This research was supported by Ministry of Education and Science of Russia (Agreement 14.613.21.0018 from 22.10.2014, RFMEFI61314X0018).

\section{References}

[1] Zhirabok, A. \& Usoltsev, S. (2001). Linear methods for fault diagnosis in nonlinear systems, Proc. European Control Conf. ECC'01, Porto, Portugal, pp. 1363-1366.

[2] Filaretov, V.F.; Zirabok, A.N.; Zuev A.V. \& Protsenko, A.A. (2012). The Development of the Faults Accommodation System for Actuators of Multilink Manipulator, Proc. of the 23rd International DAAAM Symposium, Zadar, Croatia, pp.575-578.

[3] Filaretov, V.F.; Zhirabok, A.N.; Zuev, A.V. \& Procenko, A.A. (2013). Synthesis Method of Fault Tolerant Control System for Manipulators, Journal of Advanced Material Researches. Vol. 717. pp. 551-556.

[4] Isermann, R. (2006). Fault-Diagnosis Systems: An Introduction from Fault Detection to Fault Tolerance, SpringerVerlag: Berlin, pp. 475.

[5] Shumsky, A.Ye. \& Zhirabok, A.N. (2006). Nonlinear diagnostic filter design: Algebraic and geo-metric points of view, Int. J. Applied Mathematics and Computer Science, vol. 16, no . 1, pp. 115-127.

[6] Frank, P. (1990). Fault diagnosis in dynamic systems using analytical and knowledge-based redundancy - A survey and some new results, Automatica, vol. 26, pp. 459-474.

[7] Staroswiecki, M.; Yang, H. \& Jiang, B. (2006). Progressive accommodation of aircraft actuator faults, Proc. IFAC Symp. Safeprocess'2006, Beijing. China, pp. 877-882.

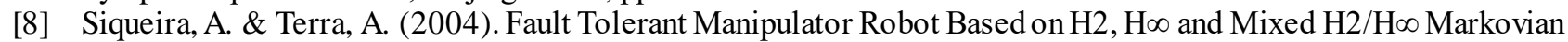
Controls, Proc. of the IEEE International Conference on Control Applications, Taipei, Taiwan, pp. 309-314.

[9] Filaretov, V.; Zhirabok, A. \& Kucher, D. (2008). New approach to robust observer design, Proc. 19-th Intern. DAAAM Symposium, Slovakia, pp.491-492.

[10] Filaretov, V.F. (2007). Synthesis of Adaptive Control Systems for Electric Servo Actuators of Manipulators, Proc. of the 18th DAAAM Int. Symp. «Intelligent Manufacturing \& Automation», Zadar, Croatia, pp. 277 - 279. 